RESEARCH ARTICLE

\title{
Machine-learning guided optimization of laser pulses for direct-drive implosions
}

\author{
Fuyuan Wu(1,2,‡, Xiaohu Yang ${ }^{2,3, \ddagger}$, Yanyun Ma ${ }^{2,3}$, Qi Zhang ${ }^{1,2}$, Zhe Zhang ${ }^{4}$, Xiaohui Yuan ${ }^{1,2}$, \\ Hao Liu ${ }^{1,2}$, Zhengdong Liu ${ }^{5}$, Jiayong Zhong ${ }^{2,5}$, Jian Zheng ${ }^{2,6}$, Yutong Li ${ }^{2,4}$, and Jie Zhang ${ }^{1,2}$ \\ ${ }^{1}$ Key Laboratory for Laser Plasmas (MOE) and School of Physics and Astronomy, Shanghai Jiao Tong University, Shanghai 200240, \\ China \\ ${ }^{2}$ Collaborative Innovation Center of IFSA, Shanghai Jiao Tong University, Shanghai 200240, China \\ ${ }^{3}$ Department of Physics, National University of Defense Technology, Changsha 410073, China \\ ${ }^{4}$ Beijing National Laboratory for Condensed Matter Physics, Institute of Physics, Chinese Academy of Sciences, Beijing 100190, China \\ ${ }^{5}$ Department of Astronomy, Beijing Normal University, Beijing 100875, China \\ ${ }^{6}$ Department of Plasma Physics and Fusion Engineering, University of Science and Technology of China, Hefei 230026, China \\ (Received 17 November 2021; revised 24 December 2021; accepted 24 January 2022)
}

\begin{abstract}
The optimization of laser pulse shapes is of great importance and a major challenge for laser direct-drive implosions. In this paper, we propose an efficient intelligent method to perform laser pulse optimization via hydrodynamic simulations guided by the genetic algorithm and random forest algorithm. Compared to manual optimizations, the machine-learning guided method is able to efficiently improve the areal density by a factor of $63 \%$ and reduce the in-flight-aspect ratio by a factor of $30 \%$ at the same time. A relationship between the maximum areal density and ion temperature is also achieved by the analysis of the big simulation dataset. This design method has been successfully demonstrated by the 2021 summer double-cone ignition experiments conducted at the SG-II upgrade laser facility and has great prospects for the design of other inertial fusion experiments.
\end{abstract}

Keywords: double-cone ignition; genetic algorithm; pulse optimization; random forest

\section{Introduction}

Laser-driven fusion ${ }^{[1-3]}$ is a promising approach for the realization of controlled fusion energy. Many laser-driven fusion schemes have been proposed in the past decades, including the indirect-drive central ignition ${ }^{[4-6]}$, the directdrive central ignition ${ }^{[7-9]}$, the hybrid-drive ignition ${ }^{[10-12]}$, the cone-guided fast ignition ${ }^{[13-16]}$, the shock ignition ${ }^{[17,18]}$, the impact ignition ${ }^{[19]}$ and the double-cone ignition $(\mathrm{DCI})^{[20]}$. In the DCI scheme, the conventional central ignition process is replaced by four progressive processes: quasi-isentropic compression, acceleration, collisional preheating and magnetic field assisted fast ignition. The requirements for the

Correspondence to: J. Zhang, Key Laboratory for Laser Plasmas (MOE) and School of Physics and Astronomy, Shanghai Jiao Tong University, Shanghai 200240, China. Email: jzhang1@sjtu.edu.cn

${ }^{\ddagger}$ Fuyuan Wu and Xiaohu Yang contributed equally to this work. drive laser energy can be reduced significantly because the compression and acceleration of fuels are performed in two head-on gold cones. The need for fast ignition laser energy can also be relaxed to an affordable level of the current PW laser technology with head-on collisional preheating and magnetic field collimated fast electrons.

However, one of the challenges remains in the optimization of the drive laser pulse to obtain a desired implosion with high areal density, high ion temperature and small in-flightaspect ratio (IFAR) with a given drive energy. At least three shocks should be controlled precisely to achieve a high areal density with relatively small IFAR. This requires to efficiently optimize 22 independent parameters of the laser pulse in a vast parameter space.

With the rapid development of supercomputers and data science, machine learning has been successfully applied in laser fusion research ${ }^{[21-26]}$. For example, 

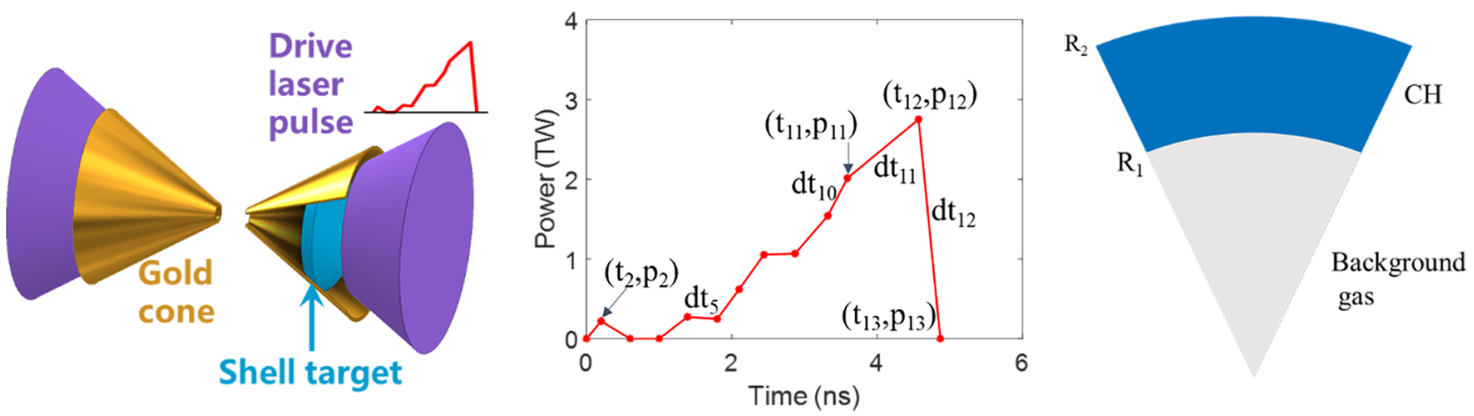

Figure 1. Schematic of the DCI target structure and laser pulse to be optimized in this paper.

Gopalaswamy et al. ${ }^{[23]}$ established the relationship between 1D hydrodynamic simulations and 3D experiment data by using Bayesian inference, leading to a tripled increase of neutron yield at the OMEGA laser facility. Humbird et al. ${ }^{[24]}$ used transfer learning to train the deep neural network with simulation data and experiment data, greatly improving the prediction ability of the calculation model. Peterson et al. ${ }^{[25]}$ used the random forest algorithm to build a surrogate model based on 60,000 2D HYDRA simulations and discovered a new class of robust implosion at the National Ignition Facility (NIF). Rose et al. ${ }^{[26]}$ used the genetic algorithm to optimize the laser pulse at the OMEGA laser facility and obtained an increased neutron yield.

In this paper, we propose a machine-learning method with the combination of the genetic algorithm and random forest algorithm to guide hydrodynamic simulations for the design of the drive laser pulse in the DCI scheme. The 1D code MULTI-IFE ${ }^{[27,28]}$ is employed to efficiently determine the areal density and ion temperature corresponding to a given laser pulse under spherical symmetry approximation. This intelligent approach is not only suitable for the DCI scheme, but is also applicable to other laser drive fusion schemes. For example, if the laser pulse is replaced with an X-ray pulse, this method can be used to optimize the X-ray pulse shape for laser indirect-drive experiments.

This paper is organized as follows. Section 2 describes the details of the optimization method. Section 3 presents the optimization results and demonstrates the effectiveness of the method with preliminary DCI experimental results. Finally, a summary is given in Section 4.

\section{Method}

A schematic of the DCI target structure and the drive laser pulse is illustrated in Figure 1. The target is made of a $\mathrm{CH}$ plastic shell $\left(\mathrm{C}_{16} \mathrm{H}_{16}\right)$. The target structure described by the inner radius $R_{1}$ and the outer radius $R_{2}$ remains unchanged in this paper, although it can be included as an independent variable. The laser pulse shape is described by 13 points. The first point is used to define the start of the laser pulse, with its time $t_{1}$ and power $p_{1}$ set to 0 . The last point is used to define the end of the pulse with its power $p_{13}$ set to 0 . The penultimate point is used to define the peak of the pulse, with its power $p_{12}$ set to the maximum power allowed by the laser facility. In total, there are 22 independent parameters $\left(\left[t_{2}, \ldots, t_{13} ; p_{2}, \ldots, p_{11}\right]\right)$. These parameters can vary freely in a huge parameter space, which makes it time-consuming to find an optimal pulse shape manually. Typically, at least 2 weeks would be needed to find an optimal pulse for the experimental campaign. In order to optimize the pulse duration flexibly, the time intervals between adjacent points $\left(\left[\mathrm{d} t_{1}, \mathrm{~d} t_{2}, \ldots, \mathrm{d} t_{12}\right]\right)$ rather than the time of each point $\left(\left[t_{2}, t_{3}\right.\right.$, $\left.\left.\ldots, t_{13}\right]\right)$ are used to describe the laser pulse shape.

The laser pulse optimization for a given drive energy can be written as follows:

$$
\left\{\begin{array}{l}
{\left[\rho, T_{\mathrm{i}}, \mathrm{IFAR}\right]=f\left(\mathrm{~d} t_{\text {laser }}, p_{\text {laser }}\right),} \\
\sum_{i=1}^{i=12} 0.5\left(p_{i}+p_{i+1}\right) \mathrm{d} t_{i} \leq E_{\text {laser }},
\end{array}\right.
$$

where $\rho$ is the peak areal density, $T_{\mathrm{i}}$ is the peak ion temperature, IFAR is the peak IFAR, $\mathrm{d} t_{\text {laser }}$ and $p_{\text {laser }}$ are the time intervals and powers, $\mathrm{d} t_{i}$ and $p_{i}$ are the $i$ th time interval and laser power, respectively, and $E_{\text {laser }}$ is the drive laser energy.

During the optimization, we pursue a comprehensive implosion performance with a well-balanced high areal density, high ion temperature and small IFAR. The fitness evaluation function used by the genetic algorithm is constructed as follows:

$$
\text { fitness }=k_{1} \rho+k_{2} T_{\mathrm{i}}-k_{3} \mathrm{IFAR} / 40,
$$

where $\rho$ is the shell areal density in $\mathrm{g} / \mathrm{cm}^{2}, T_{\mathrm{i}}$ is the shell ion temperature in $\mathrm{keV}$, IFAR is the peak IFAR of the plasma shell, and $k_{1}, k_{2}$ and $k_{3}$ are weight coefficients between 0 and 1 . The negative sign before the IFAR implies that a small IFAR is preferred during the optimization.

The simulation dataset obtained by each round of genetic algorithm optimization is analyzed with the regression model of the random forest algorithm. The feature importance of each independent variable can be extracted by the random forest algorithm and utilized to set the initial laser pulse shape of the next round of optimization. Typically, three to five rounds of genetic 

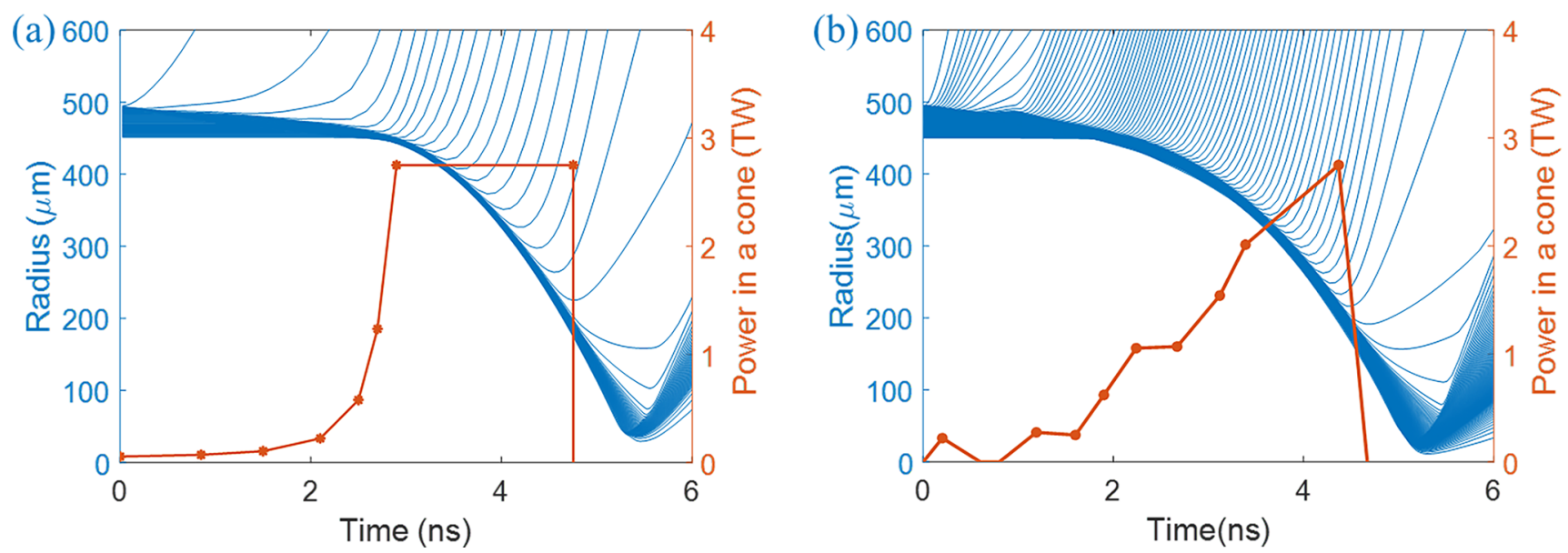

Figure 2. Plasma implosion diagram and laser pulses obtained by (a) manual optimization and (b) machine-learning optimization.

algorithm optimizations are needed to obtain a candidate laser pulse shape for the experiments.

\section{Results}

\subsection{One-dimensional optimization guided by the genetic algorithm}

Figure 2 shows the target implosion trajectory and the laser pulse shape obtained by manual optimization and machinelearning optimization. The target is made of $\mathrm{CH}$ with an inner radius of $450 \mu \mathrm{m}$ and a thickness of $45 \mu \mathrm{m}$. The manually optimized laser pulse is carefully tuned according to the requirements of isentropic implosion with a cost of 2 weeks during the 2020 winter DCI campaign. The laser pulse is then optimized under the same constraints, except that the drive energy is reduced from 12 to $10 \mathrm{~kJ}$ and the laser contrast is reduced from 100 to 15 for non-zero points. The minimum time intervals between two adjacent points are set as $200 \mathrm{ps}$ based on the arbitrary waveform generator (AWG) tuning precision of the SG-II upgrade laser facility.

The code MULTI-IFE ${ }^{[27,28]}$ used in the simulations is a 1D hydrodynamics program with separated temperatures for electrons and ions, a multi-group for radiation transport and a fusion package. The fusion reaction is turned off during the simulations. The equations of state and opacity are taken from the models of MPQEOS ${ }^{[29]}$ and SNOP $^{[30]}$, respectively.

It can be seen that the laser pulses are similar overall because both of them meet the requirements of quasiisentropic compression, which needs low powers at an early time to launch weak shocks and high powers at a late time to generate higher ablation pressure. However, some new features appear in the machine-learning optimized pulse and can be interpreted as follows. More points are used to describe the laser pulse so that the launch and propagation of the shocks can be controlled more precisely. Thirteen points are used to define the laser pulse on the balance of shocks control and pulse implementation at the SG-II
Table 1. Typical properties of manual optimization and machinelearning optimization.

\begin{tabular}{lcc}
\hline Properties & Manual optimization & Machine learning optimization \\
\hline$\rho\left(\mathrm{g} / \mathrm{cm}^{2}\right)$ & 0.38 & 0.62 \\
$T_{\mathrm{i}}(\mathrm{keV})$ & 0.25 & 0.23 \\
$V(\mathrm{~km} / \mathrm{s})$ & 233 & 233 \\
IFAR & 35 & 28 \\
Energy $(\mathrm{kJ})$ & $2 \times 6$ & $2 \times 5$ \\
\hline
\end{tabular}

upgrade facility. The low power foot at the beginning is replaced by a picket as a result of the reduced laser contrast and the preference of a small IFAR. A ramp rather than a flat top is used at the end of the pulse to better match the increase of material pressure during implosion.

The implosion performances before and after machinelearning optimization are shown in Table 1. It can be seen that the areal density has been increased by $63 \%$ (from 0.38 to $0.62 \mathrm{~g} / \mathrm{cm}^{2}$ ) because the shock sequence is better controlled so that the $\mathrm{CH}$ shell stagnates closer to the spherical center. The implosion velocity decreases a little (from 233 to $223 \mathrm{~g} / \mathrm{cm}^{2}$ ) due to the reduced drive energy. Besides, the IFAR is also reduced by $30 \%$ (from 35 to 28 ) due to the better control of the shock sequence and higher adiabat on the target outer surface. A better control of the shock sequence means the shocks are launched by the laser pulse at a proper time and break out the target inner surface at a proper radius. In the genetic algorithm optimized implosion, a moderate picket at $0.2 \mathrm{~ns}$ is employed to set a relatively high adiabat on the target surface while keeping the shock strength satisfying the requirement of isentropic compression. It is suggested to keep the IFAR smaller than 30 to mitigate the development of hydrodynamic instability ${ }^{[7]}$.

\subsection{Data analysis with the random forest algorithm}

In order to better understand the physical mechanism behind the genetic algorithm optimization, the typical laser pulses and individual fitness obtained by the genetic algorithm 

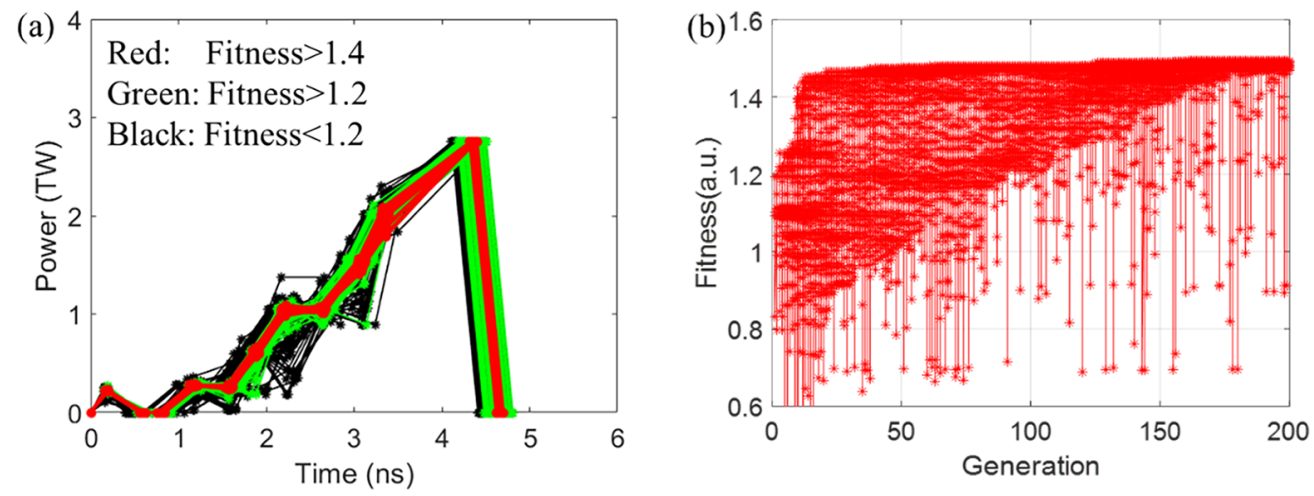

Figure 3. (a) Typical laser pulses searched by the genetic algorithm and (b) the evolution of population fitness during the optimization.

are visualized in Figure 3. The individual fitness is defined as fitness $=0.65 \rho\left[\mathrm{g} / \mathrm{cm}^{2}\right]+0.3 T_{\mathrm{i}}[\mathrm{keV}]-0.05 \mathrm{IFAR} / 40$ to obtain a high areal density and moderate ion temperature. It can be seen that the laser pulses are distributed in a wide range for early generations and converge to a narrow region later because of the genetic algorithm guidance. For the early generations, there are some laser pulses that have small drive energies because the powers are too low, or the pulses are too short. These pulses with small energies are less likely to survive during the natural selection of the genetic algorithm because they have low fitness. Due to the random gene mutation, there are always some pulse shapes corresponding to low fitness. A general tendency of the genetic algorithm is that the maximum fitness increases rapidly at an early time because the initial population has a good diversity, and then increases slowly as the average fitness approaches the maximum fitness.

The random forest algorithm is powerful in helping the genetic algorithm to jump out of local optimal trap via the analysis of feature importance. The feature importance obtained by the random forest algorithm is presented in Figure 4. It can be seen that all variables of the laser power are important, but the variables $\mathrm{d} t_{1}$ and $p_{2}$ are particularly important. This is because they describe the picket in the laser pulse and determine the first shock before the main pulse arrives. Therefore, we can modify these values $\left(\mathrm{d} t_{1}, p_{2}\right.$ and so on) to obtain the initial laser pulse for the next round of genetic algorithm optimization. The feature importance of some variables is zero, because these variables are assigned with constants during the optimization. For example, the powers $\left(p_{3}\right.$ and $\left.p_{4}\right)$ of the laser pulse are set as zero, because we found that a period of low powers is required after the relatively high-power picket, and laser pulses with powers close to zero are less reproducible at the laser facility.

Many rounds of optimization can be performed to obtain the final laser pulse, but three to five rounds are usually enough for experimental designs. One reason is that the differences of the optimal laser pulses between two rounds will become very small as the optimization converges. Another reason is that other fluctuations in experiments would be larger than these small differences due to the complexity of laser-driven implosions. In this work, the optimal pulse of the fourth round, as shown in Figure 2(b), is selected as the candidate laser pulse of the 2021 summer DCI experiments, although the shell areal density can be increased from 0.62 to $0.68 \mathrm{~g} / \mathrm{cm}^{2}$ with another round of optimization, as shown in Figure 4(b).

It is interesting to see that both the areal density and ion temperature have upper limits for a given drive energy, since there always exists some entropy increase during the implosion and the maximum imploding velocity is limited by the hydrodynamic instability (IFAR). In Figure 4(b), the upper border of the $T_{\mathrm{i}}-\rho$ scatter can be fitted as $T_{\mathrm{i}}=$ $0.62 /(1+\rho)^{2}$, where $T_{\mathrm{i}}$ is the shell ion temperature in $\mathrm{keV}$ and $\rho$ is the peak areal density in $\mathrm{g} / \mathrm{cm}^{2}$. This implies that we cannot obtain an implosion with high temperature and areal density at the same time. The final goal to achieve a high ion temperature or areal density depends on the preference of the ignition scheme. For fast ignition schemes, implosions with high areal density and moderate ion temperature are preferred, since the ion temperature will be greatly increased by the heating of fast electrons.

Although the final optimized laser pulse of the genetic algorithm depends on the choice of $k_{1}, k_{2}$ and $k_{3}$ in the fitness function fitness $=k_{1} \rho+k_{2} T_{\mathrm{i}}-k_{3} \mathrm{IFAR} / 40$, which determines whether an individual will survive or not during the natural selection, the relationship $T_{\mathrm{i}}=0.62 /(1+\rho)^{2}$ in Figure 4(b) has little dependence on the choice of the fitness function. The reason is that most kinds of laser pulses are searched and evaluated during the random optimization guided by the genetic algorithm at given laser energy and intensity.

\subsection{Demonstration with DCI experimental results}

The above machine-learning design method, a combination of machine-learning and hydrodynamic simulations, has been demonstrated by the 2021 summer DCI experiments 

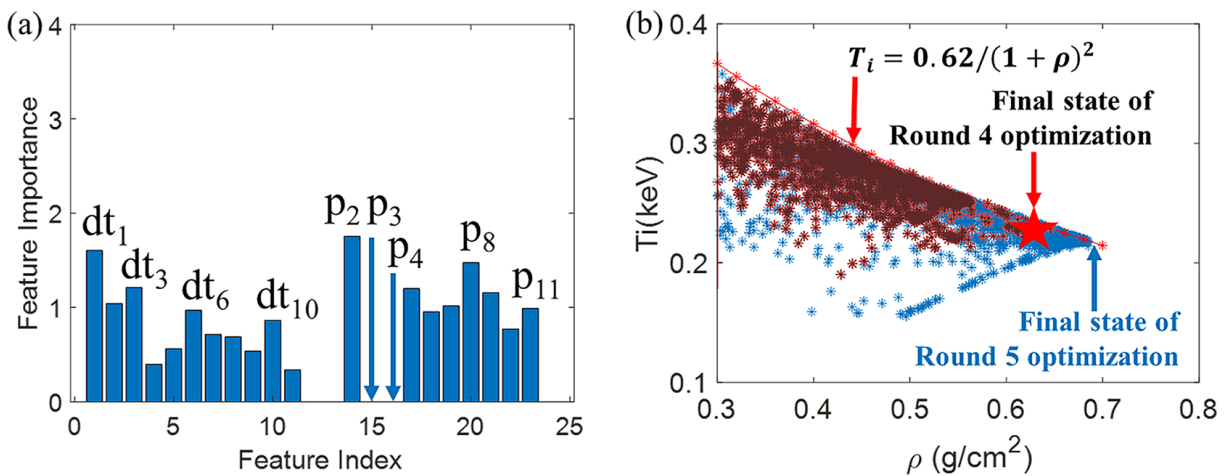

Figure 4. (a) Feature importance obtained by the random forest algorithm and (b) scatter diagram of the ion temperature and the areal density for the last two rounds of optimization.
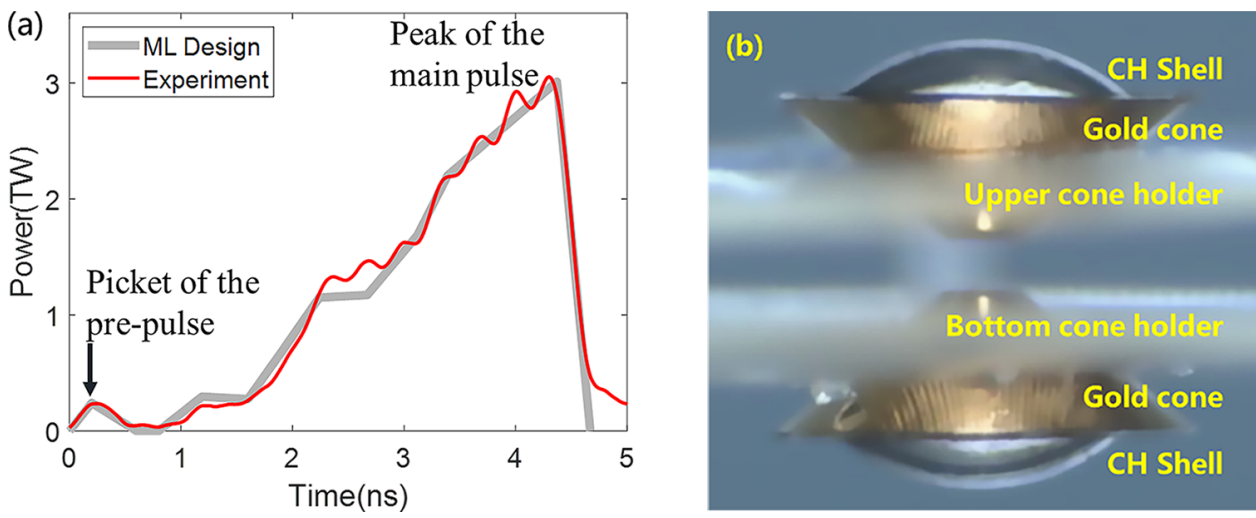

Figure 5. (a) Typical laser pulse power in a cone and (b) double-cone target used in the 2021 DCI summer experiments.

conducted at the SG-II upgrade facility. The typical laser pulse and double-cone target are presented in Figure 5. The laser pulse is implemented by overlapping four beams with a total energy of $5 \mathrm{~kJ}$ on one side. It can be observed that the machine-learning designed laser pulse can be well realized at the SG-II upgrade facility, including the time intervals and laser powers of the pulse. The double-cone target is made of a $\mathrm{CH}$ shell, gold cone and target holder. The $\mathrm{CH}$ shell has an inner radius of $450 \mu \mathrm{m}$ and a thickness of $45 \mu \mathrm{m}$. The gold cone has a thickness of $20 \mu \mathrm{m}$ and an open angle of $100^{\circ}$.

Although the detailed experimental results are being interpreted and will be published in other papers, preliminary analysis indicates that the main experimental results are in good agreement with the machine-learning simulations, as shown in Table 2. For example, the measured imploding velocity before collision is about $210 \mathrm{~km} / \mathrm{s}$, the peak ion temperature during the collision is about $200 \mathrm{eV}$ and the time cost is about $0.85 \mathrm{~ns}$. These agreements imply that the main part of the $\mathrm{CH}$ shells implodes spherically in the gold cone, although there exist high-dimensional perturbations caused by hydrodynamic instabilities and non-uniform laser irradiance.

The experimental results for the 2020 winter DCI campaign designed with the manual optimization method
Table 2. Comparison of the predicted and observed results in the DCI experimental campaign.

\begin{tabular}{lccc}
\hline Properties & $\begin{array}{c}\text { 2021 summer } \\
\text { simulations }\end{array}$ & $\begin{array}{c}\text { 2021 summer } \\
\text { measurements }\end{array}$ & $\begin{array}{c}\text { 2020 winter } \\
\text { measurements }\end{array}$ \\
\hline$T_{\mathrm{i}}(\mathrm{eV})$ & 230 & $200 \pm 50$ & $165 \pm 50$ \\
$V_{\text {imp }}(\mathrm{km} / \mathrm{s})$ & 223 & $210 \pm 25$ & $135 \pm 25$ \\
$t_{\text {cost }}(\mathrm{ns})$ & 0.60 & $0.85 \pm 0.2$ & $1.19 \pm 0.2$ \\
$\rho\left(\mathrm{g} / \mathrm{cm}^{2}\right)$ & 0.62 & $0.20 \pm 0.1$ & $0.13 \pm 0.08$ \\
\hline
\end{tabular}

(Figure 2(a)) are also included in Table 2. It can be observed that the experimental results are indeed improved significantly with the machine-learning optimizations. The physical mechanism may be the laser-plasma instability, and hydrodynamic instabilities are better suppressed with the machine-learning designed laser pulse. Further analysis of the experimental results is under the way and will be published elsewhere.

However, it should be noted that some differences exist between the predictions and measurements. The biggest difference is that the simulated areal density is smaller than the measured areal density. One reason is that the simulated result is time-resolved while the experimental result is timeintegrated. If the simulated areal density is averaged by the 'moving average method' with a time period of 200 ps, 
the simulated areal density will decrease to $0.56 \mathrm{~g} / \mathrm{cm}^{2}$ and become closer to the measurements. The other reason is that the areal density is more sensitive to the perturbations caused by the laser pulse shape, hydrodynamic instabilities and implosion asymmetry. A better match between the simulations and measurements may be achieved by optimizing the laser pulse power towards a more robust pulse shape with a higher $\mathrm{CH}$ shell adiabat or replacing the 1D hydrodynamics simulations with 2D simulations in the future.

\section{Conclusions}

A machine-learning approach is proposed for the optimized design of pulse shapes in laser drive fusion experiments based on efficient hydrodynamic simulations guided by the genetic algorithm and random forest algorithm. The optimization period of laser pulse shapes for an experiment campaign could be reduced from 2 weeks to 2 days with significant higher areal density (an increase of 63\%) and smaller IFAR (a decrease of 30\%) under similar laser conditions. The efficiency and effectiveness of this method have been well demonstrated with the 2021 summer campaign of the DCI experiments conducted at the SG-II upgrade laser facility.

In the future, we will use this intelligent method to design laser pulses and target structures for the upcoming DCI experiments with larger drive laser energies. The hydrodynamic instabilities and implosion asymmetry will also be considered and optimized with an upgrade version of the MULTI-2D program, so that a more predictive optimization can be achieved.

\section{Acknowledgments}

This work was supported by the Strategic Priority Research Program of Chinese Academy of Sciences (Nos. XDA25051200 and XDA25050200) and Startup Fund for Young Faculty at SJTU (No. 21X010500627).

\section{References}

1. N. Basov, P. Kriukov, S. Zakharov, Y. Senatsky, and S. Tchekalin, IEEE J. Quantum Electron. 4, 864 (1968).

2. J. Nuckolls, L. Wood, A. Thiessen, and G. Zimmerman, Nature 239, 139 (1972).

3. J.-L. Miquel and E. Prene, Nuclear Fusion 59, 032005 (2018).

4. O. Hurricane, D. Callahan, D. Casey, P. Celliers, C. Cerjan, E. Dewald, T. Dittrich, T. Doppner, D. Hinkel, and L. B. Hopkins, Nature 506, 343 (2014).

5. A. Zylstra, A. Kritcher, O. Hurricane, D. Callahan, K. Baker, T. Braun, D. Casey, D. Clark, K. Clark, and T. Doppner, Phys. Rev. Lett. 126, 025001 (2021).

6. A. B. Zylstra, O. A. Hurricane, D. A. Callahan, A. L. Kritcher, J. E. Ralph, H. F. Robey, J. S. Ross, C. V. Young, K. L. Baker, D. T. Casey, T. Döppner, L. Divol, M. Hohenberger, S. Le Pape, A. Pak, P. K. Patel, R. Tommasini, S. J. Ali,
P. A. Amendt, L. J. Atherton, B. Bachmann, D. Bailey, L. R. Benedetti, L. Berzak Hopkins, R. Betti, S. D. Bhandarkar, J. Biener, R. M. Bionta, N. W. Birge, E. J. Bond, D. K. Bradley, T. Braun, T. M. Briggs, M. W. Bruhn, P. M. Celliers, B. Chang, T. Chapman, H. Chen, C. Choate, A. R. Christopherson, D. S. Clark, J. W. Crippen, E. L. Dewald, T. R. Dittrich, M. J. Edwards, W. A. Farmer, J. E. Field, D. Fittinghoff, J. Frenje, J. Gaffney, M. Gatu Johnson, S. H. Glenzer, G. P. Grim, S. Haan, K. D. Hahn, G. N. Hall, B. A. Hammel, J. Harte, E. Hartouni, J. E. Heebner, V. J. Hernandez, H. Herrmann, M. C. Herrmann, D. E. Hinkel, D. D. Ho, J. P. Holder, W. W. Hsing, H. Huang, K. D. Humbird, N. Izumi, L. C. Jarrott, J. Jeet, O. Jones, G. D. Kerbel, S. M. Kerr, S. F. Khan, J. Kilkenny, Y. Kim, H. Geppert Kleinrath, V. Geppert Kleinrath, C. Kong, J. M. Koning, J. J. Kroll, M. K. G. Kruse, B. Kustowski, O. L. Landen, S. Langer, D. Larson, N. C. Lemos, J. D. Lindl, T. Ma, M. J. MacDonald, B. J. MacGowan, A. J. Mackinnon, S. A. MacLaren, A. G. MacPhee, M. M. Marinak, D. A. Mariscal, E. V. Marley, L. Masse, K. Meaney, N. B. Meezan, P. A. Michel, M. Millot, J. L. Milovich, J. D. Moody, A. S. Moore, J. W. Morton, T. Murphy, K. Newman, J.-M. G. Di Nicola, A. Nikroo, R. Nora, M. V. Patel, L. J. Pelz, J. L. Peterson, Y. Ping, B. B. Pollock, M. Ratledge, N. G. Rice, H. Rinderknecht, M. Rosen, M. S. Rubery, J. D. Salmonson, J. Sater, S. Schiaffino, D. J. Schlossberg, M. B. Schneider, C. R. Schroeder, H. A. Scott, S. M. Sepke, K. Sequoia, M. W. Sherlock, S. Shin, V. A. Smalyuk, B. K. Spears, P. T. Springer, M. Stadermann, S. Stoupin, D. J. Strozzi, L. J. Suter, C. A. Thomas, R. P. J. Town, E. R. Tubman, C. Trosseille, P. L. Volegov, C. R. Weber, K. Widmann, C. Wild, C. H. Wilde, B. M. Van Wonterghem, D. T. Woods, B. N. Woodworth, M. Yamaguchi, S. T. Yang, and G. B. Zimmerman, Nature 601, 542 (2022).

7. S. Atzeni and J. Meyer-ter-Vehn, The Physics of Inertial Fusion: Beam Plasma Interaction, Hydrodynamics, Hot Dense Matter (Oxford University Press, Oxford, 2004), Vol. 125.

8. V. Goncharov, T. Sangster, T. Boehly, S. Hu, I. Igumenshchev, F. Marshall, R. McCrory, D. Meyerhofer, P. Radha, and W. Seka, Phys. Rev. Lett. 104, 165001 (2010).

9. R. Ramis, B. Canaud, M. Temporal, W. J. Garbett, and F. Philippe, Matter Radiat. Extremes 4, 055402 (2019).

10. X. He, J. Li, Z. Fan, L. Wang, J. Liu, K. Lan, J. Wu, and W. Ye, Phys. Plasmas 23, 082706 (2016).

11. K. Lan, J. Liu, Z. Li, X. Xie, W. Huo, Y. Chen, G. Ren, C. Zheng, D. Yang, and S. Li, Matter Radiat. Extremes 1, 8 (2016).

12. X. He, High Energy Density Phys. 36, 100804 (2020).

13. M. Tabak, J. Hammer, M. E. Glinsky, W. L. Kruer, S. C. Wilks, J. Woodworth, E. M. Campbell, M. D. Perry, and R. J. Mason, Phys. Plasmas 1, 1626 (1994).

14. R. Kodama, P. Norreys, K. Mima, A. Dangor, R. Evans, H. Fujita, Y. Kitagawa, K. Krushelnick, T. Miyakoshi, and N. Miyanaga, Nature 412, 798 (2001).

15. Z. Sheng, Y. Sentoku, K. Mima, J. Zhang, W. Yu, and J. Meyerter-Vehn, Phys. Rev. Lett. 85, 5340 (2000).

16. W.-M. Wang, P. Gibbon, Z.-M. Sheng, and Y.-T. Li, Phys. Rev. Lett. 114, 015001 (2015).

17. R. Betti, C. Zhou, K. Anderson, L. Perkins, W. Theobald, and A. Solodov, Phys. Rev. Lett. 98, 155001 (2007).

18. R. Scott, K. Glize, L. Antonelli, M. Khan, W. Theobald, M. Wei, R. Betti, C. Stoeckl, A. G. Seaton, and T. Arber, Phys. Rev. Lett. 127, 065001 (2021).

19. M. Murakami, H. Nagatomo, T. Johzaki, T. Sakaiya, A. Velikovich, M. Karasik, S. Gus' Kov, and N. Zmitrenko, Nuclear Fusion 54, 054007 (2014). 
20. J. Zhang, W. Wang, X. Yang, D. Wu, Y. Ma, J. Jiao, Z. Zhang, F. Wu, X. Yuan, and Y. Li, Philos. Trans. R. Soc. A 378, 20200015 (2020).

21. P. Hatfield, S. Rose, and R. Scott, Phys. Plasmas 26, 062706 (2019).

22. P. W. Hatfield, J. A. Gaffney, G. J. Anderson, S. Ali, L. Antonelli, S. B. du Pree, J. Citrin, M. Fajardo, P. Knapp, and B. Kettle, Nature 593, 351 (2021).

23. V. Gopalaswamy, R. Betti, J. Knauer, N. Luciani, D. Patel, K. Woo, A. Bose, I. Igumenshchev, E. Campbell, and K. Anderson, Nature 565, 581 (2019).

24. K. D. Humbird, J. L. Peterson, B. Spears, and R. G. McClarren, IEEE Trans. Plasma Sci. 48, 61 (2019).
25. J. L. Peterson, K. Humbird, J. E. Field, S. T. Brandon, S. H. Langer, R. C. Nora, B. K. Spears, and P. Springer, Phys. Plasmas 24, 032702 (2017).

26. S. Rose, P. Hatfield, and R. Scott, Philos. Trans. R. Soc. A 378, 20200014 (2020).

27. R. Ramis and J. Meyer-ter-Vehn, Comput. Phys. Commun. 203, 226 (2016).

28. F. Wu, R. Ramis, Z. Li, Y. Chu, J. Yang, Z. Wang, S. Meng, Z. Huang, and J. Ning, Fusion Sci. Technol. 72, 726 (2017).

29. A. Kemp and J. Meyer-ter-Vehn, Nucl. Instrum. Methods Phys. Res. Sect. A 415, 674 (1998).

30. K. Eidmann, Laser Particle Beams 12, 223 (1994). 